ESTUDIOS / RESEARCH STUDIES

\title{
LA AGENDA NATURALISTA DE LAS PASIONES EN LA FILOSOFÍA DE LA MODERNIDAD TEMPRANA
}

\author{
Leonel Toledo Marín \\ Universidad Autónoma de la Ciudad de México \\ leontoledo@gmail.com \\ ORCID iD: https://orcid.org/0000-0002-3990-7385 \\ Samuel Herrera-Balboa \\ Universidad de La Frontera, Chile \\ samuel.herrera@ufrontera.cl \\ ORCID iD: https://orcid.org/0000-0002-0509-2907 \\ Carmen Silva \\ Universidad Nacional Autónoma de México \\ carmensilva55@gmail.com \\ ORCID iD: https://orcid.org/0000-0002-2933-8158
}

Recibido: 5 julio 2016; Aceptado: 10 enero 2018.

Cómo citar este artículo/Citation: Toledo Marín, Leonel; Herrera-Balboa, Samuel y Silva, Carmen (2018). "La agenda naturalista de las pasiones en la filosofía de la modernidad temprana”, Asclepio, 70 (1): p216. https://doi.org/10.3989/asclepio.2018.09

RESUMEN: En este artículo trataremos de caracterizar las principales razones teóricas del cambio de perspectiva del escolasticismo a la filosofía de la modernidad temprana en lo concerniente al estudio de las facultades cognitivas y emotivas. Para lograr nuestro objetivo, sintetizaremos el contexto intelectual del estudio de las pasiones; después, distinguiremos dos grandes corrientes del pensamiento naturalista: en primer lugar, la tesis reduccionista que fue adoptada, entre otros, por Thomas Hobbes, Pierre Gassendi y René Descartes; en segundo lugar, el proyecto de establecer y describir la "dinámica de la vida mental" que fue desarrollado por Thomas Hobbes, John Locke y David Hume. Al dar cuenta de esto, esperamos también obtener una comprensión más clara sobre los cambios de perspectiva que fueron propuestos por algunos filósofos de la modernidad temprana, cuyas ideas avanzaron hacia la naturalización de la antropología filosófica.

PALABRAS CLAVE: Naturalismo; Pasiones; Antropología naturalizada; Filosofía moderna temprana.

\section{THE NATURALISTIC AGENDA OF PASSIONS IN EARLY MODERN PHILOSOPHY}

ABSTRACT: In this paper we will try to characterize the main theoretical grounds that promoted the change of view from Scholasticism to Early Modern Philosophy on the emotive and cognitive faculties; to achieve this task, we will summarize the intellectual background for the study of passions; then, we will differentiate two mainly "naturalistic" trends of thought: First, the reductionist thesis, adopted by Thomas Hobbes, Pierre Gassendi and René Descartes, among others; and second, the attempt to establish and describe the dynamics of mental life, which was developed by Thomas Hobbes, John Locke and David Hume. On giving this account, we expect also to gain a clearer comprehension about the changes of perspective that were proposed by some Early Modern thinkers, whose views developed towards a new naturalized philosophical anthropology.

KEY WORDS: Naturalism; Passion; Naturalized anthropology; Early modern philosophy 


\section{EL CONTEXTO INTELECTUAL DEL ESTUDIO DE LAS PASIONES}

Con tal de tener un marco general desde el cual establecer nuestras reflexiones, a continuación esquematizaremos tres grandes rutas por las cuales se estudiaron las pasiones desde la antigüedad. Por supuesto, debemos advertir que cada una de éstas posee una historia compleja, y dar cuenta de cada una de ellas está más allá de los límites que nos proponemos en este trabajo.

La primera ruta de investigación la constituye la tradición iniciada por los escritos de Galeno (129216) (Nutton, 1995, pp. 79-80; Nutton, 2004, pp. 230-247) quien definió dos importantes áreas de indagación que no dejaron de causar tensiones a lo largo de la historia:

Por un lado, Galeno analizó las relaciones entre la filosofía y la medicina, considerando sus diferencias y sus mutuas críticas. ${ }^{1}$ Debemos señalar que la actitud galenista ante la filosofía de sus contemporáneos es de reproche frente a lo que él considera excesos y descuidos metodológicos. Un ejemplo de esto lo encontramos en el siguiente pasaje:

"Es preciso, pues, que vigilemos lo que dicen los filósofos actuales. Pero es mejor no decir 'filósofos', pues si practicaran la filosofía cuidarían en primer lugar lo siguiente: sacar los principios de sus demostraciones a partir de los fenómenos evidentes. Y esto los antiguos lo hicieron más que ninguna otra cosa, y por ello fueron llamados sabios entre los hombres. No escribieron tratados, ni basaron las demostraciones en la teoría dialéctica o natural, sino que, sacando los principios de las virtudes de los propios fenómenos evidentes, ejercieron las virtudes con sus acciones y no con sus palabras". (Galeno, 2003, pp. 201-202)

Todavía más, la crítica de Galeno en el tratado Las facultades del alma siguen los temperamentos del cuerpo, no se limita a sus contemporáneos, sino que se extiende a los problemas e inconsistencias que él encuentra en las teorías platónicas y aristotélicas del alma racional, las cuales intenta refutar con fenómenos y observaciones. Ahora bien, estas críticas no le impiden a Galeno caracterizar al médico como un auténtico filósofo, pues la medicina comprende todas las partes de la filosofía:

"Así pues, ¿qué es lo que falta todavía para que el médico que practica el arte en un modo digno de Hipócrates no sea filósofo? Pues si para desentrañar la naturaleza del cuerpo, las diferencias entre las enfermedades y los remedios indicados le conviene haberse ejercitado en la especulación lógica, y para perseverar diligentemente en el ejercicio de estas cosas haber despreciado la riqueza y cultivado la moderación, abarcaría ya todas las partes de la filosofía: lógica, física y ética". (Galeno, 2002a, p. 90)

Por otro lado, la investigación galenista se ocupó del análisis de la naturaleza del alma ${ }^{2}$ y de sus facultades considerando una terapéutica enmarcada por la teoría de los humores, sus expresiones en el temperamento y sus enfermedades. Éstas últimas entendidas como el desequilibrio de algún elemento en el cuerpo (Isidoro de Sevilla, 2004, pp. 475-476) o, también, debidas a alguna causa externa como los fenómenos meteorológicos o influencias desde el nivel supralunar. Además, tal teoría supuso una clasificación del carácter y sus padecimientos, así como sus manifestaciones en la moral. Esta tradición se prolongó en los tratados árabes de medicina que fueron traducidos en el s. XII (Conrad, 1995, pp. 92-125) hasta el análisis de las enfermedades del alma -como la melancolía- durante los siglos XVI y XVII. ${ }^{3}$

En estrecho vínculo histórico y temático con la tradición médica, la segunda ruta que nos interesa indicar es la que se dedica a la descripción del alma, de sus potencias y de la caracterización de sus facultades en distintos niveles, particularmente el gnoseológico (Aristóteles, 2000, pp. 414a29-414b20). Esta segunda vía está constituida por la abundante exégesis sobre el De anima de Aristóteles, llevada a cabo por los filósofos árabes. Al-Farabi, Avicena y Averroes establecieron, por ejemplo, distintas teorías acerca de las facultades del alma ${ }^{4} \mathrm{y}$, posteriormente, los escolásticos formularon sus propias tesis al respecto (Hasse, 2014, pp. 305-319). A lo largo de dicha exégesis se constituyó un complejo esquema que partía de las bases de la percepción compartidas por seres humanos y animales (Aristóteles, 2000, pp. 434b30-435a) hasta las potencias superiores que eran exclusivamente humanas. Una breve descripción de estas potencias la encontramos en Tomás de Aquino:

"Mas para el conocimiento perfecto del sentido, que sea suficiente al animal, se requieren cinco cosas. En primer lugar, que el sentido reciba la especie del sensible, ${ }^{5}$ y esto pertenece al sentido propio. Segundo, que juzgue acerca de los sensibles percibidos y los discierna entre sí; lo cual es preciso que se haga por la potencia a la cual llegan todos los sentidos, y que se denomina sentido común. Lo tercero es que las especies recibidas de los sensibles se conserven, pues el animal necesita de la aprehensión de los sensibles no sólo en su presencia, sino también después que hayan desaparecido; y esto es necesario que se atribuya a otra potencia, pues también en las cosas 
corporales es distinto el principio de recibir y el de conservar [...] y esta potencia se llama imaginación o fantasía". (Tomás de Aquino, 2001a, p. 561)

Hasta aquí, esta descripción del proceso de la operación de las facultades sensibles es común a los seres humanos y a los animales. Después de estas bases, los elementos serán depurados y analizados de distinto modo: encontraremos ahora intenciones y se establecerán las primeras diferencias entre los seres instintivos y los que poseen "potencia cogitativa". En palabras del Aquinate:

"En cuarto lugar, se requieren algunas intenciones que el sentido no aprehende, como lo nocivo o lo útil, y otras cosas parecidas; y el hombre llega a conocer estas cosas investigando y comparando, mientras que los otros animales lo hacen por cierto instinto natural [...] por eso, a esto se ordena la estimativa natural en los otros animales, y en el hombre la potencia cogitativa, que es comparativa de las intenciones particulares, por lo cual también se llama razón particular y entendimiento pasivo. En quinto lugar, se requiere que aquellas cosas que fueron aprehendidas antes por el sentido y se conservan interiormente, se traigan de nuevo para una consideración actual; y esto pertenece a la potencia de la memoria, que en los otros animales tiene su operación sin investigación, y en los hombres con indagación y estudio: por lo cual en los hombres no sólo es memoria sino reminiscencia [...]". (Tomás de Aquino, 2001a, p. 561) ${ }^{6}$

Según la descripción medieval, el ser humano posee las facultades naturales y también el intelecto. ${ }^{7}$ Éste sería jerárquicamente superior a las facultades naturales y tiene como función abstraer las especies inteligibles (Tomás de Aquino, 2001b, pp. 776-778). Además, se encarga de las actividades cognitivas asociadas al conocimiento universal-científico, de las operaciones lógicas y de la voluntad (Tomás de Aquino, 2001b, pp. 773-796). Entre otras características, "la mente era inmortal, no localizada en ningún órgano corporal y le concernían el conocimiento de los universales, como el lenguaje o la habilidad de razonar lógicamente". (Kemp y Fletcher, 1993, p. 565)

Además de las dos rutas ya señaladas anteriormente, encontramos una tercera vía para abordar las pasiones: la tradición retórica. Ésta tiene como fuente el texto aristotélico y su desarrollo propio en los pensadores latinos: la conexión entre las pasiones y los sentimientos encontraron en la política y también en las bellas artes su aplicación más importante (Aristóteles, 2002, pp. 6-7). Advertimos que la riqueza y la complejidad de la retórica en el estudio de las pasiones merece una investigación aparte, y señalemos que los tropos literarios, los recursos estilísticos del discurso, así como las técnicas artísticas en la pintura, la escultura, la poesía, la música y el teatro fueron estudiados rigurosamente a lo largo de los Ss. XVI-XVIII dentro de la tradición retórica, para lograr los efectos deseados en el público.

Debemos indicar aquí que cada una de estas tres rutas evolucionó a lo largo de la Edad Media en la medida en que sus desarrollos se volvieron más sofisticados. Pero, al menos desde las formulaciones de las tesis galenistas hasta la concepción medieval, el estudio de las pasiones fue incorporado en el corpus total del saber. Una vez más, tengamos en cuenta, como se ha visto con Galeno, que el área de conocimiento que podía resumir estas tres vertientes fue la medicina, en el sentido de que ésta comprendía el dominio de todas las artes liberales. Tal y como lo expone Isidoro de Sevilla:

“1. [...] En efecto, el médico debe conocer la gramática, para poder entender y exponer lo que lee. 2 . Lo mismo cabe decir de la retórica, de modo que pueda delimitar con argumentos indiscutibles los casos que tiene entre manos. Otro tanto hay que afirmar de la dialéctica, que le permite, mediante el raciocinio, profundizar en las causas que provocan las enfermedades y en los remedios aplicables para su curación. Necesita de la aritmética, por lo que se refiere al número de horas que duran los ataques febriles y la periodicidad que presentan. 3. Digamos lo mismo de la geometría, en cuanto a la índole de las regiones o zonas en las que señala qué es lo que cada uno debe observar. $E$ incluso no debe ignorar la música, pues muchas son las enfermedades que, como puede leerse en los libros, han sido tratadas utilizando esta disciplina [...] 4. Conocerá, en fin, también la astronomía, [...] Pues, como sostiene algún médico, al par de las variaciones que se van presentando, nuestro cuerpo experimenta igualmente alteraciones. 5. De aquí que se considere a la medicina como una segunda filosofía. Una y otra ciencia reclaman para sí al hombre entero; pues si por una se sana el alma, por la otra se cura el cuerpo". Etimologías (Isidoro de Sevilla, 2004, pp. 495-497).

Entonces, pese a la aparente autonomía de estas tres vías de investigación sobre las facultades emotivas durante un largo período de la historia intelectual, cada una de las rutas se incorporó en un esquema más general del conocimiento, cuyo ideal fue la relación orgánica de todas las disciplinas y donde la expresión más elevada de este ideal de scientia se resumía en la filosofía o en la medicina. Si esto puede decirse del estudio de las pasiones durante la Edad Media, entonces, la pregunta que intentaremos resolver es: ¿en qué medida se puede caracterizar la transformación 
de la investigación relativa a las pasiones durante la modernidad temprana?

Nuestra hipótesis es que a diferencia de la gran revolución cosmológica -que se ha entendido como el cambio más o menos claro del punto de vista ptolemaico al sistema copernicano- las teorías sobre las pasiones cambiaron de maneras más complejas durante la modernidad temprana. Sostenemos que tal transformación consistió en un doble desarrollo: primo, implicó la reducción ontológica y la simplificación de los patrones explicativos en torno a los procesos fisiológicos y psíquicos del ser humano. Secundo, encontramos un paulatino movimiento de síntesis de las tres rutas de conocimiento que hemos indicado arriba, para convertirse en una sola.

Todo lo anterior dio lugar a una "nueva" ciencia de la naturaleza humana donde las pasiones jugaron un papel central. Este transcurso de la historia intelectual bien puede entenderse como una serie de pasos hacia la naturalización de la antropología filosófica, que llegará a constituir una nueva agenda de investigación para las facultades emotivas y cognitivas. Esta forma diferente de abordar las pasiones tendrá repercusiones en los tratados ilustrados, en las tesis darwinianas y en la psicología contemporánea. A continuación, trataremos de dar cuenta de algunos momentos fundamentales de este periodo de la historia de las ideas.

\section{REDUCCIÓN Y SÍNTESIS DE LAS FACULTADES EMO- TIVAS Y COGNITIVAS: LA PSICOLOGÍA MECANICISTA}

Algunos estudiosos de la modernidad temprana, tales como Gary Hatfield (Hatfield, 2012, pp.151186; Hatfield, 1998, pp. 988-990) y Gábor Boros (Boros, 2006, pp.125-142), coinciden en que uno de los cambios característicos de la nueva perspectiva fue la adopción del esquema mecanicista y el rechazo del hilemorfismo en la comprensión de la fisiología y la psicología humanas. Éste es un cambio que se desarrolla paralelamente a los descubrimientos de algunos elementos centrales para la comprensión futura del sistema nervioso y sus implicaciones en las diversas funciones del organismo. El conocimiento más preciso de la anatomía cerebral humana que se logró a partir de las observaciones realizadas por Andrés Vesalio (1514-1564) (Wear, 1995, pp. 215-361), entre otros, tornó obsoleta la visión galénica de los ventrículos cerebrales asociados a las facultades cognitivas (Kemp y Fletcher, 1993, p. 566). Esto motivó a los filósofos naturales a revisar más cuidadosamente el modo en que el sistema nervioso pudiera constituir una suerte de vasos comunicantes entre la información sensorial y las facultades cognitivas más complejas.

\subsection{La tesis Hobbes-Gassendi}

Desde sus escritos tempranos redactados durante la década de 1620 a 1630, Thomas Hobbes y Pierre Gassendi ya habían asumido que el variado esquema de las facultades puede reducirse todavía más a los distintos movimientos (voluntarios e involuntarios) derivados de procesos fisiológicos (Sorell, 1993, pp. 235272). Estos filósofos sostuvieron, por un lado, que las facultades de percepción y las facultades superiores relativas al conocimiento pueden reducirse a un par: la imaginación, según el filósofo francés, ${ }^{8}$ y las operaciones mentales propias del cálculo para el inglés; ${ }^{9}$ por otro, el complejo panorama de las pasiones en Hobbes puede reducirse al deseo de permanecer vivo $y$, en Gassendi, al deseo fundamental de la felicidad, entendida como una forma de placer, en el marco de una ética epicúrea. ${ }^{10}$

Para Hobbes y Gassendi, el estudio de las pasiones debe hacerse considerando que las facultades intelectuales mismas son una manifestación más de las facultades naturales, análogas a las que podemos encontrar en todos los animales. Podría parecer que estos filósofos se adhieren al esquema medieval de las potencias que atribuye a los animales ciertas facultades análogas al razonamiento, tal y como lo hemos visto en el pasaje del Aquinate. Sin embargo, debajo de esta apariencia debemos reconocer la tensión que provoca la propuesta Hobbes-Gassendi frente al esquema tradicional. Más bien, en esta tesis hay una clara intención de reducir las facultades superiores -que eran inmateriales en el esquema medieval- al mismo funcionamiento mecánico y, por tanto, material o natural (Paganini, 2002, pp. 20-41). En otras palabras, desde la perspectiva de estos autores tenemos un continuo entre todos los seres vivos, o si se prefiere, una homogenización entre los animales y los hombres con relación a sus facultades. Recordemos que esta posición se opone a la versión más tradicional de la filosofía renacentista y moderna, según la cual el ser humano se encuentra por encima del resto de los seres creados.

La asunción anterior tiene como corolario la siguiente tesis: siendo el razonamiento un producto de la fisiología mecánica, los animales muestran en mayor o menor grado, algún tipo de raciocinio. La propuesta que antepone Gassendi a René Descartes en sus "Quintas objeciones" y en su Disquisitio metaphysica (duda V, Inst.III, Dubitatio VI) puede sinte- 
tizarse del siguiente modo: si tanto en el ser humano como en los animales podemos encontrar órganos sensoriales, nervios y espíritus animales que se conectan con el cerebro, entonces podemos aceptar que los animales poseen también la estructura fisiológica propia de la fantasía o imaginación y, por tanto, ¿por qué no plantear lo mismo para la razón? Pensar, como lo hacen Descartes y la tradición, que las operaciones racionales distintivas del alma son "superiores", queriendo decir con ello "inmateriales", no es más que jugar con las palabras; así, Gassendi le hace notar a Descartes: "el hecho de que tú te apliques especialmente la denominación de 'espíritu' puede constituir una denominación de una naturaleza más noble, pero no por esto de una naturaleza diferente" (Gassendi, 1964, pp. 150-151).

En efecto, es posible que los animales no posean razonamiento ni pasiones al modo humano, pero eso no quiere decir, afirma Gassendi, que no las posean a su modo y que debamos negarles esas facultades:

“Dices: 'Los animales no poseen razón'. Ellos carecen de una razón humana, pero no de aquella que les es propia, de tal manera que parece que no se puede decir que estén privados de razón sino con relación a nosotros y a nuestra especie [...] Pero si sus razonamientos no son tan perfectos y no los conducen a tantas cosas como a los seres humanos, aun con ello razonan, y parece que no hay nada de diferente [en ellos], sino sólo en lo que se refiere a más o menos". (Gassendi, 1964, pp. 152-153)

Además, al reducir las facultades intelectuales a las facultades meramente naturales, Gassendi apunta en su argumentación hacia una dirección tal vez inesperada: el razonamiento termina asociado a la misma base fisiológica que durante la tradición se le atribuyó al placer y al dolor, a las preferencias y decisiones, al miedo y a la felicidad. La sugerencia implícita es que el intelecto ahora puede ser analizado con el mismo esquema materialista que el de las pasiones.

Para el caso de la filosofía hobbesiana, una vez establecidos los principios de la ciencia del movimiento de los cuerpos, debe continuarse con las emociones, que funcionan como los movimientos de la vida psíquica:

"De la física hay que pasar a la Moral, en la que se consideran los movimientos de las mentes, como el apetito, la aversión, el amor, la benevolencia, la esperanza, el miedo, la ira, la emulación, la envida, etc., qué causas tienen y de qué cosas sean causa ellas mismas, porque hay causas que proceden de los sentidos y de la imaginación, y que son objeto de estudio de la Física". (Hobbes, 2000, p. 80)
Ciertamente, el movimiento y el contacto físico de las partes de los cuerpos en el nivel sensorial compone, a su vez, la base de los procesos perceptuales. Estos parten sucesivamente del orden de las imágenes obtenidas por los cinco sentidos (Hobbes, 2000, pp. 300-307), después al nivel del placer y el dolor (Hobbes, 2000, p. 308), luego la deliberación, la voluntad y la libertad (Hobbes, 2000, pp. 309-310), a continuación el nivel racional y el conocimiento científico (Hobbes, 1997, pp. 31-36) y, finalmente, al orden de las pasiones. ${ }^{11}$ Esta descripción materialista de la psicología animal y humana establece la continuidad y la reducción de todos sus niveles funcionales a procesos fisiológicos. ${ }^{12}$ En Hobbes, al igual que en Gassendi, las operaciones intelectuales superiores no implican la inmaterialidad. La caracterización hobbesiana del proceso que va de la imaginación al entendimiento es ilustrativa:

"La imaginación que surge en el hombre (o en cualquier otra criatura dotada con la facultad de imaginar), a través de las palabras o por otros signos voluntarios es lo que generalmente llamamos entendimiento, y es común al hombre y al animal".

(Hobbes, 1997, p. 19)

En la tesis Hobbes-Gassendi, la diferencia que hay entre las operaciones intelectuales más complejas del ser humano y aquellas que muestran los animales, no es una diferencia ontológica, sino de grado de desarrollo y especificidad de funciones.

\subsection{La tesis Hobbes-Gassendi en Descartes}

En tensión con su propia metafísica dualista, Descartes en su tratado de Las pasiones del alma (1649), se ve impulsado a asumir la perspectiva naturalista de Hobbes y de Gassendi, acercándose a la idea de que el alma y sus funciones pueden ser concebidas en una relación indisoluble con el arreglo y la disposición material del cerebro. Descartes caracteriza las pasiones del siguiente modo: "Pueden definirse en general como percepciones, sentimientos o emociones del alma que se refieren particularmente a ella y que son causadas, mantenidas y fortalecidas por algún movimiento de los espíritus [animales]." (Descartes, 1997, pp. 95-96; AT XI, p. 349)..$^{13}$

La lectura de Las pasiones... indica que la clave explicativa de Descartes en este proceso es el movimiento de los espíritus animales a través de distintas redes de transporte de los impulsos. Éstos van desde las terminales nerviosas ubicadas en los órganos sensoriales hasta la glándula pineal, en el centro del cerebro. En la perspectiva que se adopta en esta obra, notamos 
que la psicología cartesiana se muestra poco preocupada por mantener su dualismo sustancial y mucho más dedicada a las descripciones mecánicas y fisiológicas de las causas de las pasiones. En este sentido, la reducción ontológica parece anunciarse: en lugar de contar con los diversos niveles de mediación típicamente escolásticos (p. ej., las especies sensibles e inteligibles, el sentido común o las facultades sensoriales o el intelecto pasivo y activo), los procesos fisiológicos y cognitivos se simplifican y se resuelven en uno solo, tal y como queda detallado en el "Ejemplo de la manera en que las impresiones de los objetos se unen en la glándula que está en medio del cerebro":

“Por ejemplo, si vemos a un animal venir hacia nosotros, la luz reflejada de su cuerpo dibuja dos imágenes de él, una en cada uno de nuestros ojos; esas dos imágenes forman otras dos, por medio de los nervios ópticos, en la superficie interior del cerebro; después de ahí, mediante los espíritus animales de los que están llenas las cavidades, aquellas pequeñas imágenes irradian de tal suerte hacia la pequeña glándula rodeada por los espíritus, que el movimiento que compone cada punto de una de las imágenes tiende hacia el mismo punto de la glándula [...] la cual, actuando inmediatamente contra el alma, le hace ver la figura de ese animal". (Descartes, 1997, p. 110; AT XI, p. 355)

Evidentemente, para los estándares contemporáneos esta hipótesis resulta poco clara y problemática, ${ }^{14}$ pero veamos más bien la estrategia cartesiana. Allí hay un intento por mejorar la versión escolástica cuando se hace intervenir en la explicación sólo a las imágenes, que son transportadas directamente al centro del cerebro. Además, tomemos en cuenta la simplificación en el nivel de las facultades: el sentido común, la memoria, los intelectos pasivo y activo, son comprendidos en una sola facultad intelectiva que tiene relación -aunque no esclarecida- con la glándula pineal.

El proceso descrito por Descartes continúa según las siguientes líneas:

\footnotetext{
"Y, además, si esa figura es muy extraña y espantosa, es decir, si tiene estrecha relación con las cosas que antes han sido perjudiciales para el cuerpo, provoca en el alma la pasión del temor y, después, la del arrojo, o bien la del miedo o el espanto, según el diferente temperamento del cuerpo o la fuerza del alma y según como uno se haya preparado antes, mediante la defensa o la huida, contra las cosas perjudiciales con las que se relaciona la impresión presente". (Descartes, 1997, p. 111; AT XI, p. 356)
}

La pasión, entonces, es primeramente una respuesta ante una imagen o ante la información que se recibe en el cerebro a través de los espíritus animales. Di- cha respuesta puede variar según la propia fisiología o según una suerte de entrenamiento a través del cual puede modificarse. Al aceptar esa posibilidad, Descartes se acerca todavía más a una descripción de las pasiones en términos de una psicología naturalista, y esto es explícito en la preparación de la respuesta de huida o defensa. Tal entrenamiento, según Descartes:

\begin{abstract}
“...organiza el cerebro de algunos hombres de tal modo que los espíritus reflejados de la imagen así formada sobre la glándula desde ahí se trasladan, en parte, a los nervios que sirven para volver la espalda y mover las piernas para huir $y$, en parte, a los que ensanchan o estrechan de tal manera los orificios del corazón; o bien que agitan de tal modo las otras partes de donde se le envía sangre, la cual, ratificándose de forma distinta de la acostumbrada, envía al cerebro espíritus apropiados para mantener y fortalecer la pasión del miedo, es decir, apropiados para tener abiertos, o bien para abrir de nuevo, los poros del cerebro que los conducen a los mismos nervios".

(Descartes, 1997, p. 111; AT XI, p. 356)
\end{abstract}

Hemos dicho que la hipótesis cartesiana parecería que ha tomado en serio las sugerencias de Hobbes y de Gassendi acerca de que el alma misma puede ser el producto de la organización del sistema nervioso y que, entonces, el alma siendo material, podría sentir y pensar. El resultado de la fórmula es ahora patente en tanto que las pasiones son explicadas en términos de las respuestas a nivel fisiológico, en una cadena causal mecánica. Una vez establecido este principio, la descripción de las pasiones puede avanzar hacia la especificidad de cada una de las respuestas, pero todas ellas operan bajo el mismo supuesto y la misma descripción básica.

\section{EL DESARROLLO POSTERIOR DE LAS TESIS NATURALISTAS}

\subsection{El peso de la tradición y el programa reduccionista}

Si bien tanto Descartes como Gassendi usaron argumentos que dirigen el rumbo hacia la naturalización completa del mundo psíquico, no mantuvieron siempre una reducción absoluta de las operaciones anímicas a procesos exclusivamente cerebrales o fisiológicos. ${ }^{15}$ Ambos pensadores cedieron ante sus propios marcos teológicos y religiosos, y no fueron capaces de abrazar la radicalidad de la tesis hobbesiana de las pasiones para avanzar hacia una versión más simple de las facultades cognitivas y emotivas. ${ }^{16}$

Particularmente, el problema que se encuentra en la versión gassendiana y la cartesiana de las pasiones es la tensión provocada entre su descripción natura- 
lista y el carácter superior del alma, en su dimensión inmaterial. He aquí un problema que impide a veces sopesar la novedad de las teorías de los dos filósofos para el siglo XVII. En efecto, por un lado, en su Tratado de las pasiones, "al mecanizar las funciones del alma sensitiva, incluyendo las operaciones cognitivas que están en la base del instinto y la memoria, concebidas ahora como ocurriendo sin la prisión mental, Descartes, efectivamente, creó una psicología mecánica" (Hatfield, 2012, p. 181). ${ }^{17}$ Algo similar podría decirse de Gassendi y Hobbes.

Sin embargo, esta misma descripción ligada a la asunción del alma inmaterial, puede ser interpretada como una mera continuidad de la tesis aristotélica, como lo hace Peter R. Anstey: "Pues en lo que toca a la descripción del alma dada por el Descartes histórico, está en muchos temas tanto histórica como conceptualmente ligada a la de Aristóteles" (Anstey, 2000, p. 255). Todavía más, en contraste con esta postura de la mera continuidad con la tradición, encontramos asunciones como la de Sarah Byers para quien, en Descartes y en Gassendi podemos atestiguar sólo ignorancia y empobrecimiento de la tesis aristotélica, al reducir anima al mero movimiento local y a procesos meramente mecánicos:

"Los argumentos con los que Descartes buscó rebatir el alma vegetativa descansaban sobre un sentido errado del "movimiento". Más aún, entre los objetores contemporáneos de Descartes que, igual que Descartes, atacaron la postura aristotélica de que la vida requería anima [...] no encontramos algún indicio de haber entendido lo que Aristóteles quiso decir con "la vida es auto-movimiento". (Byers, 2006, p. 754). ${ }^{18}$

Nuestra interpretación es distinta, pues creemos que el resultado que se deja entrever es, en todo caso, una agenda de investigación sobre la razón asociada naturalmente a las pasiones. Una agenda que está basada en la descripción mecánica-fisiológica que tiene una extensión o un correlato psicológico, de tal modo que, como afirma Descartes, "la naturaleza parece haber unido cada movimiento de la glándula a cada uno de nuestros pensamientos desde el comienzo de nuestra vida, y el hábito puede [además], unirlos a otros de forma similar" (Descartes, 1997, p. 127; AT XI, p. 368).

A la luz de esta concepción moderna, la base fisiológica de la vida psíquica -incluyendo en ella las facultades que tradicionalmente se habían asociado con el intelecto- se convirtió en el cimiento sobre el que se construiría toda la sociedad y, en este sentido, la vida política. ${ }^{19}$ Sobre esta última es conveniente señalar junto con Harold J. Cook, que:

\footnotetext{
"Teorías explícitamente republicanas pudieron haber sido soterradas, Descartes pudo haber sido docilitado, y Hobbes (como Maquiavelo y Spinoza) pudieron haberse convertido en nombres sinónimos de condenación. Pero el intento de extraer conclusiones sobre el cuerpo político a partir de las nuevas nociones del cuerpo natural ha permanecido con nosotros, para la mayor consternación de aquellos que desean encontrar la virtud en el poder de la razón aparejada con lo inmanente o lo trascendente". (Cook, 2002, p. 47)
}

La asunción explícita de esta propuesta significó el giro propiamente moderno del análisis de las facultades cognitivas y, con ello, de las pasiones. Podemos sostener que dicha transformación derivó en la antropología filosófica naturalista que sustituyó, paulatinamente, las preocupaciones y los dogmas teológicos de la filosofía escolástica tradicional.

Lo cierto es que el problema de la glándula pineal o de la comunicación de las sustancias y la sugerencia de que la materia puede pensar, trasminó rápidamente del ámbito de las posturas filosóficas hacia la discusión entre los médicos. Este fue un elemento que avivó las querellas sobre la naturaleza del alma y su relación con el cuerpo que eran sostenidas entre los seguidores de Galeno, Paracelso y Jean Batista van Helmont, entre otros. ${ }^{20}$ Fue en esa área donde se elaboraron las teorías tanto a favor como en contra de asociar a la razón humana con las pasiones, así como de emparentar a la especie humana con la de los animales. (Thomson, 2010, pp. 3-37)

\subsection{Otro sentido para el naturalismo: la dinámica de las pasiones}

Si la primera vía de investigación relativa a las pasiones desembocó en la búsqueda de las bases fisiológicas de las facultades cognitivas junto con las emotivas, la segunda se dedicó a configurar una nueva ciencia a partir de la descripción de dichas facultades con herramientas conceptuales referidas exclusivamente a lo mental. Veamos en qué sentido Hobbes se compromete con esta otra perspectiva. Según Bernard Gert:

"Una vez que Hobbes llega a los conceptos de apetito y aversión, placer y dolor, su explicación de las pasiones individuales ignora completamente la relación entre la conducta humana y su filosofía materialista. Él simplemente continúa por la vía de la introspección y la experiencia, usando libremente ideas de la explicación aristotélica de las pasiones [...] Para Hobbes, es muy claro que la introspección y la experiencia, y no la filosofía materialista, proveen 
la clave para la comprensión de la conducta humana". (Gert, 1996, pp. 160-161)

En efecto, las propuestas hobbesianas acerca del conocimiento del mundo psíquico incluyen el criterio de la experiencia propia, derivada de la asunción de que tal mundo es común para la especie humana. Así, el conocimiento de los hombres es descriptible en términos de "Nosce te ipsum, conócete a ti mismo", lo que para el filósofo inglés implica:

“[... ] Enseñarnos que, a partir de la similitud de los pensamientos y las pasiones de un hombre con los pensamientos y pasiones de otro, quienquiera que se observe a sí mismo y que considere lo que hace cuando piensa, opina, razona, espera, teme, etcétera, y sobre qué fundamentos lo hace, con ello él aprenderá y sabrá cuáles son los pensamientos y las pasiones de todos los otros hombres en ocasiones similares". (Hobbes, 1997, p. 10)

De ahí que, cuando las emociones han sido descritas como movimientos análogos a los que se producen en la materia, la tarea pendiente consiste en establecer y formular una dinámica propia para las pasiones que sea aplicable a todos los seres humanos. Dicha dinámica de la vida mental es fundada bajo los mismos principios derivados del aspecto funcional mecanicista. En este sentido, el mecanicismo deja de ser un supuesto ontológico para convertirse en un modelo explicativo de la vida psíquica.

Además, esta nueva dinámica de las pasiones se formula añadiendo una característica metodológica esencial, vale decir, que sus criterios de análisis y demostración están basados en la experiencia tanto interna como externa. El recurso metodológico de la experiencia que hemos apuntado, en parte, es el que había caracterizado a la tradición médica. Aunque una discusión sobre los distintos conceptos de experiencia que tienen lugar en la modernidad temprana excede los límites de este trabajo, podemos mencionar como un ejemplo del intento de dirimir esta cuestión, el texto de Charles Wolfe (Wolfe, 2010, pp. 335 y ss.) donde se reconoce al menos tres vías de discusión en torno a la experiencia y a la tradición empirista: el experimentalismo de la Royal Society, el empirismo moral de John Locke y David Hume y, finalmente, un empirismo de raíz médica sostenido por William Harvey, Pierre Gassendi, Thomas Sydenham, junto con los vitalistas de Montpellier.

Nuestra interpretación acerca de la experiencia referida a la nueva dinámica de las pasiones se restringe a tres sentidos que están en íntima conexión:
1. La recolección de datos y de fenómenos que pueden ser relevantes en la investigación. Esta acepción posee una antigua raíz médica expresada en la necesidad de establecer una historia de los síntomas que afectan al paciente para poder indicar una terapéutica apropiada. En este caso, los datos de la experiencia son signos o síntomas indicativos de alguna causa que está operando en el nivel fisiológico (Waldow, 2010, pp. 287-308).

2. La experiencia interna como introspección, reflexión u observación de las propias facultades cognitivas y emotivas en sus diversas funciones y operaciones (Locke, 1979, pp. 105-106). El supuesto indispensable de este tipo de observación es que sus resultados son extrapolables a la totalidad de los seres humanos. Ésta es una manera por la cual la experiencia interna sobrepasa el límite de lo particular para poder encontrar datos universales $y$, por tanto, con pretensiones de certeza más sólida.

3. La historia natural como recopilación y organización de datos de los fenómenos y efectos del mundo al modo baconiano y su correlato con las facultades mentales. Esto deriva en la recolección de datos de las operaciones del intelecto, de las pasiones y de la conducta humana. ${ }^{21}$

En síntesis, es propiamente una confluencia compleja del modelo mecanicista de la vida psíquica junto con el concepto de experiencia antes referido, lo que configura esta nueva dinámica experimental de las pasiones. Tal descripción de lo mental adquiere, entonces, su propio discurso y sus propios marcos conceptuales. Esa es justamente la ruta de análisis que seguirán John Locke, Baruch Spinoza y, muy particularmente, David Hume (Schmitter, 2012, pp. 255-278).

Locke y Hume fueron conscientes de que sus análisis de lo mental no requieren de la existencia del mundo material. Por ejemplo, el autor del Ensayo sobre el entendimiento humano establece desde el inicio de su obra los linderos de su agenda de estudio del siguiente modo:

"[...] siendo mi propósito investigar sobre el origen, la certeza y la extensión del conocimiento humano, junto con los fundamentos y grados de la creencia, la opinión y el asentimiento, no me entrometeré ahora con las consideraciones físicas ${ }^{22}$ sobre la mente, ni me problematizaré examinando en qué consiste su esencia, o por qué movimientos de nuestros espíritus ${ }^{23}$ o alteraciones de nuestros cuerpos llegamos a tener sensaciones a través de nuestros órganos o cualquier 
idea en nuestros entendimientos, o si esas ideas dependen o no de la materia. Éstas son especulaciones que, aunque curiosas y amenas, evitaré, en tanto que no tienen relación con el designio que ahora poseo". (Locke, 1979, p. 43)". ${ }^{24}$

En el pasaje anterior, vemos que la perspectiva lockeana suspende el juicio acerca de la tesis Hobbes-Gassendi que hemos caracterizado líneas arriba. El médico inglés también adoptará la misma actitud en su discusión acerca de la posibilidad de que la materia pueda pensar: "Tenemos las ideas de la materia y el pensamiento, pero posiblemente nunca podremos saber si algún ser exclusivamente material piense o no" (Locke,1979, p. 540). Esta tesis trasluce no sólo el escepticismo lockeano en lo respectivo a temas metafísicos (Popkin, 2003, pp. 257-261) sino que, al adoptarlo, él pudo avanzar hacia una perspectiva de investigación donde el conocimiento se define y se resuelve en el ámbito de las operaciones mentales. ${ }^{25}$ En este sentido, Locke "fue más allá tanto de Descartes como del epicureísmo contemporáneo, al sugerir una concepción unificada del ser humano" (Wright, 1991, p. 257).

David Hume, en una estrategia escéptica ${ }^{26}$ que bien recuerda al hipótesis non fingo newtoniano, anota que la tesis de la inmaterialidad del alma es tan innecesaria e indecidible para la filosofía como también lo es la asunción de su materialidad (Hume, 2000, pp.152-164). Una vez que su perspectiva queda restringida exclusivamente a lo mental, Hume parte de la distinción básica entre ideas e impresiones y de los principios de asociación (semejanza, contigüidad, causa y efecto), que funcionarán como leyes universales bajo las cuales se configuran ideas e impresiones cada vez más complejas.

Para el filósofo escocés, las pasiones son impresiones de segundo orden y están regidas por los mismos principios de asociación (Hume, 2000, pp. 185-190). A partir de esos elementos, Hume establece que aquello que llamamos el orgullo y la humildad (Hume, 2000, pp. 182-184), el vicio y la virtud, la belleza, la fealdad, (Hume, 2000, pp. 193-198), el amor y el odio (Hume, 2000, pp. 214-216), así como la simpatía y la compasión (Hume, 2000, pp. 238-240) son pasiones que comparten el mismo fundamento explicativo. La conclusión del análisis humeano es célebre: "la razón es y sólo debe ser la esclava de las pasiones, y no puede jamás pretender otro oficio que el de servirlas..." (Hume, 2000, p. 266). Así, para Hume, todo el entramado psíquico es uno solo y no hay sino facultades emotivas y cognitivas operando con las mismas leyes, válidas tanto para los seres humanos como para los animales (Hume, 2000, p. 119). ${ }^{27}$

De nuestra discusión anterior podemos sostener que la agenda de naturalización de las facultades emotivas y cognitivas posee al menos dos sentidos durante la modernidad temprana: primero, significa la adopción del esquema mecanicista en la descripción de los procesos causales-fisiológicos de las pasiones y de la razón misma, tal y como fue descrito en la tesis Hobbes-Gassendi. Segundo, se refiere al establecimiento de las leyes o principios universales y naturales con los cuales operan el mundo intelectual y emotivo.

Bajo la primera noción, la ruta que se definió vio la necesidad de encontrar y relacionar los procesos cerebrales con la actividad psíquica. Allí se entendió a las pasiones como respuestas o actividades derivadas de la interconexión con los órganos corporales. Paralelamente, la segunda vía de investigación basada en la segunda acepción no se ocupó más de la base fisiológica. Aquí, es suficiente con reconocer que las facultades emotivas y cognitivas operan en analogía con los fenómenos mecánicos. Pero la vida psíquica posee sus propios principios universales y necesarios para su actividad. ${ }^{28}$ En esta segunda vertiente de naturalización, al formular la base universal de los principios de la vida emotiva, se incluyen también los de la estética y la ética. Hume encuentra esta clave en "la fuerza de la simpatía":

\footnotetext{
"Las mentes de todos los hombres son similares en sus operaciones y sentimientos: nadie puede ser conmovido por alguna afección con la cual otros no sean susceptibles de serlo en algún grado. Como en las cuerdas uniformemente enrolladas el movimiento de una se comunica al resto, también las afecciones pasan rápidamente de una persona a otra y producen los movimientos correspondientes en cada criatura humana. Cuando veo los efectos de una pasión en el gesto y en la voz de alguna persona, mi mente inmediatamente pasa de estos efectos a sus causas, y forma una idea tan vívida de la pasión que se convierte actualmente en la pasión misma". (Hume, 2000, p. 368)
}

La postulación de estos principios constituyó una nueva ciencia concebida en analogía con los procedimientos y alcances de la ciencia física. Allí, la experiencia interna y externa es considerada como criterio de análisis y verificación. Además, ella está enmarcada en la necesidad que imponen las leyes de la geometría, tal como ocurre en los casos de Hobbes y de Spinoza, o en la necesidad newtoniana de las leyes básicas y universales del movimiento, tal como sucede con Hume. Así, dice este último: 


\begin{abstract}
"En las acciones humanas hay un curso general de la naturaleza como lo hay en las acciones del sol y del clima. Hay también caracteres peculiares a naciones distintas y personas particulares, tanto como las comunes a la humanidad. El conocimiento de tales caracteres se funda en la observación de una uniformidad en las acciones que surgen de ellos, y esta uniformidad constituye la esencia misma de su necesidad". (Hume, 2000, p. 259)
\end{abstract}

\section{A MODO DE CONCLUSIÓN}

En las páginas anteriores hemos tratado de describir cuáles fueron las transformaciones más relevantes que tuvieron lugar durante los siglos XVII-XVIII con respecto al estudio de las pasiones. Creemos que las innovaciones que se produjeron en esta época atraviesan diversas rutas teóricas que incluyen:

1. Los ecos de las discusiones que ya habían aparecido en la filosofía antigua y medieval.

2. La simplificación y reducción de las facultades reconocidas por la tradición escolástica.

3. La síntesis en una nueva concepción del ser humano y de sus facultades cognitivas y emotivas.

Los filósofos y médicos de la modernidad temprana que contribuyeron a este cambio de perspectiva lograron configurar nuevas vías de investigación sobre la vida mental. Ellos intentaron alejarse de los dogmas teológico-religiosos y extendieron la exploración de la estructura natural y cognitiva a la totalidad de la vida psicológica, así como a la vida ética, social y política.

Podemos sostener que la agenda naturalista de las pasiones durante la modernidad temprana se estableció al menos en dos agendas de investigación:

La primera es el reduccionismo naturalista. Ésta concibió una ontología mecanicista en la descripción de los procesos fisiológicos de las pasiones. Aquí hemos esclarecido los aportes de Hobbes-Gassendi y de Descartes, considerando también que abren la puerta a la explicación materialista de la vida psíquica. Y hemos planteado que tal estrategia no está exenta de tensiones ante la tradición.

Considerando esta vía reduccionista, no podemos dejar de mencionar las amplias consecuencias para el materialismo francés de la segunda parte del siglo XVII y en general para el materialismo del siglo XVIII. Aquí, según René Pintard (2000), la vía gassendiana o la vía neo-epicúrea tuvo enormes influencias en los llamados "Libertinos Eruditos". En su estudio, Pintard denomina como "tétrada libertina" al mismo Gassendi en compañía del médico Gabriel Naudé (16001653), François de La Mothe Le Vayer (1588-1672) y Élie Diodati (1566-1671).

Estos filósofos en su adherencia al corpuscularismo o al esceptismo dieron forma a un modo de enfrentar al aristotelismo que se extendió por toda la Europa ilustrada. Acompañan a estos pensadores, otros dos médicos que seguirán a Gassendi en amistad y en obra: Guy Patin (1601-1672) y François Bernier (16251688). Este último hizo el primer intento interpretativo en francés de la obra de Gassendi y es razonable sugerir que haya presentado las tesis gassendianas a su pupilo de anatomía, John Locke. ${ }^{29}$

El listado de la influencia Hobbes-Gassendi y Descartes es largo y significativo. Todos ellos asumieron un modelo reductivo de las pasiones a las estructuras fisiológicas y mecánicas de la vida psíquica e incluso política. Entre las obras que hacen eco de estas tesis podemos mencionar la descripción de la vida social expresada por las utopías de Cyrano de Bergerac (1619-1655) en Viaje a la Luna (Cyrano, 1993) y Los estados e Imperios del Sol de 1662, (Cyrano, 2003); la explicación hedonista de las relaciones sociales y morales en la Fábula de las abejas (1729), (Mandeville, 2001) de Bernard Mandeville (1670-1733); El hombre máquina (1747), (La Mettrie, 2014), y el Discurso sobre la felicidad (1748), (La Mettrie, 2005), de Julien Offray de La Mettrie (1709-1751), junto con el naturalismo fisiológico de Denis Diderot (17131784) en El sueño de D'Alembert (1769), (Diderot, 1992). También es evidente que dichas influencias continuaron en el movimiento ilustrado. En definitiva, la lectura naturalista de las pasiones hizo época y confluyó directamente en la fisiología médica y en la epistemología ilustradas.

La segunda agenda que hemos llamado la dinámica de las pasiones -cultivada por Hobbes, Locke y Hume, entre otros- se formó al sintetizar elementos intelectuales de diversa procedencia. Por un lado, tomó del mecanicismo los principios de sus analogías descriptivas: tal como las partículas materiales se combinan, las ideas y las pasiones humanas se disponen guiadas por las leyes naturales propias del entendimiento humano.

Por otro lado, los criterios de obtención de datos y de verificación para esta nueva dinámica del mundo interior se fueron situando en la necesidad y en las restricciones que impone la experiencia interna y externa. Esto derivó en una transformación radical del 
modo en que se debían estudiar las facultades cognitivas y emotivas. Los alcances de esta nueva ciencia fueron establecidos por Hume del siguiente modo:

"Debemos, por tanto, investigar nuestros experimentos en esta ciencia a partir de una observación cuidadosa de la vida humana, y tomarlos tal y como aparecen en el curso común del mundo, por la conducta de los humanos en compañía, en sus negocios o en sus recreaciones. Cuando los experimentos de este tipo sean recolectados y comparados juiciosamente, podremos esperar establecer a partir de ellos una ciencia, la cual no será inferior en certeza, y será muy superior en su utilidad a cualquier otra de comprensión humana". (Hume, 2000, p. 6)

Durante la modernidad temprana, las dos vías de investigación sobre las pasiones que hemos estudiado mostraron rápidamente su aplicabilidad y efectividad cuando se trató de establecer una tipificación inteligible y simplificada de las emociones. En la naciente psicología se postuló el paralelismo de la conducta humana con la de otros animales; en el arte del sensualismo barroco se formularon teorías bajo los principios de universalidad de las emociones, ${ }^{30}$ articuladas con el análisis de las facultades cognitivas y la retórica. ${ }^{31}$ Una aproximación más simplificada de la fisiología de las pasiones estableció los factores emotivos para entender la toma de decisiones en la política, el peso de la expresión de las emociones en el proceso de creación y recepción de toda obra artística. Dicho sea de paso, son las herramientas intelectuales del barroco con las que se aseguró la colonización de las emociones de los indígenas en el Nuevo Mundo.

Cuando establecieron las bases de la agenda naturalista de las pasiones, los filósofos de la modernidad temprana abrieron paso a las preguntas fundamentales que actualmente tienen resonancia teórica y dirigen las investigaciones contemporáneas en torno a la universalidad de las emociones, la continuidad de la expresión de éstas en las diversas especies animales ${ }^{32} y$, por supuesto, la pregunta acerca de cuáles son las emociones humanas básicas y cómo están presentes en aspectos relevantes de la cultura. Es cierto que las ideas de los pensadores que hemos presentado no poseen las sutilezas y complejidades descriptivas propias de la psicología contemporánea. Sin embargo, no deja de ser evidente que, al naturalizar la antropología, Hobbes, Gassendi, Descartes, y Hume fueron parte de la Revolución científica que cambió definitivamente el rumbo de las investigaciones sobre las pasiones y nos heredaron perplejidades que todavía están por resolverse.

\section{AGRADECIMIENTOS}

Este artículo ha sido publicado en el marco del proyecto fondecyt de iniciación no11140387 "Filosofía natural de Pierre Gassendi: fundamentos límites y horizontes para comprender las objeciones gassendistas a la filosofía cartesiana" financiado por Conicyt-Chile.

\section{NOTAS}

1. Un análisis del "eclecticismo" filosófico de Galeno lo encontramos en (Frede, 1987, pp. 290-294).

2. Sobre los temas relativos a la esencia y a la materialidad o inmaterialidad del alma, Galeno se muestra siempre agnóstico. En diversos pasajes que prefiguran por siglos la postura escéptica de los filósofos modernos John Locke y David Hume, Galeno rechaza "tener conocimiento alguno acerca de cuál sea la esencia del alma y de si es mortal e inmortal", esto porque "[...] a la hora de tratar las enfermedades, para el médico no tiene ningún interés si el alma es mortal o inmortal, así tampoco tiene interés alguno si su esencia es incorpórea, como quiere éste, o corpórea, como defiende aquél al señalar que la esencia del alma es espíritu [...]" (Galeno, 2002b, p. 336); Cfr. (Galeno, 2003, p. 174), así como en (Frede, 1987, p. 297).

3. Para un estudio sobre las preocupaciones teóricas sobre la melancolía durante el $\mathrm{s}$. XVI, remitimos al texto de Roger Bartra, Cultura y melancolía, particularmente el cap. III: "Los mitos de la melancolía y los paradigmas de la ciencia",

(Bartra, 2001, pp. 197-212). Mientras que, el locus clásico de esta tradición durante el $\mathrm{s}$. XVII sigue siendo el monumental volumen de Robert Burton, The Anatomy of Melancholy (1621); el tratamiento erudito de Burton de las facultades del alma se encuentra en Burton, 2001, pp. 154-166. Véase también Schmidt, 2004, pp. 585-592.

4. Véase para el primero, (Al-Farabi, 1995, pp. 55-67); sobre Avicena, el lector puede consultar (Avicena, 2005, pp. 2758), así como el cap. 4 del estudio de (Davidson, 1992, pp. 74-126); en el cap. 7, pp. 258-314, del mismo estudio se tratan los comentarios de Averroes al De anima aristotélico. Para una descripción comprensiva, véase también (Black, 2005, pp. 308-326)

5. Recordemos, junto con Panaccio que "la teoría de las especies floreció en la segunda mitad del Siglo Trece, pero la inspiración para ella vino de tan lejos como de Agustín y la óptica árabe". Cfr. Panaccio, 2014, p. 347. Esta teoría fue cultivada por Roger Bacon, Tomás de Aquino y, en general, por la filosofía escolástica. En la expresión de Panaccio las 
especies son "aspectos" o "semejanzas" de sí mismos que los objetos irradian continuamente y que se propagan en el medio. Las facultades sensoriales son capaces de recibir, almacenar y producir dichas especies y, a la vez, son capaces de crear nuevas a partir de las sensibles, hasta llegar a las más universales y abstractas e inmateriales, que son las especies inteligibles.

6. Las cursivas son nuestras; véase (Tomás de Aquino, 2001b, pp. 717-720).

7. (Aristóteles, 2000, pp. 134-145); así como (Al-Farabi, 1995, pp. 68-73), y (Averroes, 2004, pp. 119-142). Para una descripción específica de la noción de intelecto en los filósofos árabes y su recepción en la filosofía escolástica, véase (Black, 2014, pp. 322-331).

8. Gassendi, señala: "Asumo un alma para las semillas, propongo un alma para los animales y no hago alguna diferencia entre el entendimiento y la imaginación" (Gassendi, 1959, p. 14). Véase también, (Bloch, 1971, p. 369).

9. T. Hobbes, en Leviathan, planteará: "La razón, en este sentido, no es otra cosa sino el cálculo (esto es, sumar o sustraer) de las consecuencias de los nombres generales que acordamos para marcar y significar nuestros pensamientos. Digo marcarlos cuando calculamos por nosotros mismos, y significarlos cuando demostramos o aprobamos nuestros cálculos a otros hombres" (Hobbes, 1997, p. 32).

10. Para la cercanía de la psicología y la ética hobbesianas y gassendianas, remitimos al lector a (Sarasonhn, 1996, pp. 118-136).

11. T. Hobbes en Leviathan, Parte I, cap. VI: Of the interiour beginnings of Voluntary Motions; commonly called the Passions. And the Speeches by which they are expressed, (Hobbes, 1997, pp. 37-46). Nótese que el capítulo dedicado a las pasiones es posterior al que se dedica a la descripción de la razón

12. Para una descripción de los elementos de este proceso, véase (Gert, 1996, pp. 157-174).

13. En adelante citaremos la edición española incluida en la bibliografía y luego la edición canónica de Adam y Tannery de las CEuvres de Descartes, (Descartes, 2000, referida como AT), señalando el volumen en romanos, con su paginación en arábigas. Ejemplo: (Descartes, 1997, pp. 95-96; AT XI, p. 349).

14. Tal problematización la podemos encontrar como tesis general en Damasio (1997); Sin embargo, en contra de las apreciaciones contemporáneas desfavorables a Descartes, Gábor Boros reconoce que "fue Descartes quien desarrolló el primer tipo de neurociencia en la forma de una neuromecánica" (Boros, 2006, p. 131).

15. La posición de Gassendi en el Syntagma Philosophicum (1658) es a lo menos controversial respecto de su filosofía materialista. Bloch (1971) sostiene que la filosofía gassen- diana sería un proyecto filosófico que abandonó principios materialistas y reincorporó algunos dogmas de la fe cristiana. En el caso de Descartes, su dualismo sustancial es defendido en las Meditaciones metafísicas (1641).

16. Un estudio de la perspectiva atomista y materialista sostenida por los autores de impronta hobbesiana y gassendiana, en el contexto de las restricciones teológicas y religiosas se encuentra en (Wilson, 2010, pp. 35-51). Además, consideramos interesante destacar que esta limitante fue claramente identificada por los filósofos de la época y es acusada por Spinoza del siguiente modo: "[...] nadie, que yo sepa, ha determinado la naturaleza y la fuerza de los afectos, ni lo que puede el alma, por su parte, para moderarlos. Ya sé que el celebérrimo Descartes, aun creyendo que el alma tiene una potencia absoluta sobre sus acciones, ha intentado, sin embargo, explicar los afectos humanos por sus primeras causas, y mostrar, a un tiempo, por qué vía puede el alma tener un imperio absoluto sobre los afectos; pero, a mi parecer al menos, no ha mostrado nada más que la agudeza de su gran genio [...]" (Spinoza, 2007, p. 199).

17. Remitimos a nuestros lectores a la discusión más general acerca de las asunciones cartesianas y su recepción en la psicología contemporánea en (Hatfield, 2009, pp. 1-25).

18. Las críticas a la concepción gassendiana se encuentran en (Byers, 2006, pp. 741-748).

19. Un estudio de las implicaciones políticas del mecanicismo hobbesiano y gassendiano se encuentra en (Sarasohn, 1996, pp. 142-167).

20. Para una noticia de la variedad de prácticas y teorías médicas que estaban en debate desde diversos marcos teológicos, véase (Elmer, 1989, pp. 10-45). La manera en que las teorías del alma y de la vida mental hobbesianas y cartesianas son sometidas a la crítica, particularmente por los Platónicos de Cambridge, es discutida en (Henry, 1989, pp. 98-107).

21. Estos tres sentidos están articulados en el "Historical plain Method" de Locke, delineado en el Capítulo I, "Introducción", en (Locke, 1979). Para un estudio sobre la asunción de la historia natural en el pensamiento lockeano, véase (Anstey, 2002, pp. 65-92).

22. "Physical considerations": advirtamos que Locke usa aquí "físicas" en el sentido de una teoría de la naturaleza y, particularmente, de las teorías médicas, tal y como plantea Cook: "Physic era un arte que dependía del conocimiento impartido en la universidad. Sus practicantes eran los 'physicians', quienes eran, necesariamente, hombres con grados universitarios de física (physic): en la Inglaterra del siglo diecisiete, comúnmente con un doctorado en medicina (M.D.)" (Cook, 1990, p. 398). Entonces, las teorías que Locke está dejando de lado en su investigación son justamente las que tienen que ver con la teoría de la naturaleza de la mente, en el sentido de las tesis que han sido sostenidas por Hobbes, Gassendi y Descartes. 
23. En este pasaje, Locke hace referencia a los espíritus animales.

24. Ciertamente, hay en el trasfondo del pasaje lockeano una influencia escéptica, en resonancia con la actitud de Galeno que hemos indicado anteriormente en nuestra nota no 2. De este modo, el escepticismo también apuntala el recurso metodológico de la experiencia, en los orígenes de la nueva ciencia de las facultades cognitivas y emotivas. Al respecto, Wilson, señala lo siguiente: "Al ubicar las cosas no-percibidas, tanto corpóreas como incorpóreas fuera del ámbito del conocimiento humano práctico y eficaz, Locke estaba tratando de articular las formas de las nuevas ciencias (la natural y la moral), que no requerían de alguna referencia a entidades fuera de la experiencia. Ahora, él podría esbozar una concepción del conocimiento científico y moral basada en las premisas Epicuro-gassendianas, las cuales correspondían a lo que él consideraba la actividad real de sus héroes, particularmente Boyle en la química, Newton en las matemáticas aplicadas y Sydenham en la medicina" (Wilson, 2010, p. 50).

25. En esta vía, recordemos que para Locke el conocimiento se define como "la percepción de la conexión y el acuerdo, o de la incongruencia y el desacuerdo entre cualquiera de nuestras ideas" (Locke, 1979, p. 525).

26. Para una valoración cuidadosa de la influencia del escepticismo de la época en el pensamiento de Hume, remitimos al lector a (Popkin, 1980, pp. 103-147).

27. Los animales "actúan a partir de un razonamiento que no es en sí mismo diferente, ni fundado en principios diferentes de aquellos que aparecen en la naturaleza humana" (Hume, 2000, p. 119). Véanse también "Of the pride and humility of animals" (Hume, 2000, pp. 211-213). Y, "Of the love and hatred of animals" (Hume, 2000, pp. 255-256).

28. Es justamente este doble significado de la "naturalización de las pasiones" lo que nos separa de interpretaciones demasiado estrechas del término "naturalismo" como la de G.

\section{BIBLIOGRAFÍA}

Al-Farabi (1995), La ciudad ideal, Madrid, Tecnos.

Anstey, Peter (2000), “'De Anima' and Descartes: Making up Aristotle's Mind", History of Philosophy Quarterly, 17 (3), pp. 237-260.

Anstey, Peter (2002), "Locke, Bacon and Natural History", Early Science and Medicine, 7 (1), pp. 65-92.

Aristóteles (2000), Acerca del alma, Madrid, Editorial Gredos.

Aristóteles (2002), Retórica, Ciudad de México, Universidad Nacional Autónoma de México.

Averroes (2004), Sobre el intelecto, Madrid, Editorial Trotta.
Manning, para quien "el naturalismo científico moderno" quiere decir "brevemente, materialismo ligado a las leyes de la naturaleza" en (Manning, 2008, p. 442).

29. Acerca de la discusión sobre la influencia de Gassendi en Locke, podemos mencionar a (David Fate Norton, 1981, p. 336); y a Michael, F. y Michael, E., 1990. Otro estudio a destacar es el capítulo dedicado a la gnoseología de Gassendi en el marco de la filosofía de Locke es: (Duchesneau, 1973, pp. 93 y ss.).

30. Notemos que en ningún ideal cultural tuvo más importancia el escudriñar las pasiones en todos sus grados, combinaciones e implicaciones que en la estética barroca. Creemos que durante la modernidad temprana el estudio de la expresión de los sentimientos en los manuales de pintura, de música, y de las demás bellas artes corresponden también a un proyecto cultural en el que la retórica tiene los objetivos claros de conmover directa y eficazmente las pasiones del espectador. Cfr. (LeCoat, Gerard, (1971) pp. 207223). Un referente de este fenómeno lo constituye la obra de Charles Le Brun, Conférence sur l'expresion générale et particulière (1668), cuyo fundamento teórico es la tesis cartesiana de las pasiones. Véase (Le Brun, 1982, pp. 1-6) y (Cottegnies, 2002).

31. Estos tópicos continúan discutiéndose con descripciones cada vez más refinadas, que establecen conexiones temáticas que apuntan a la síntesis entre las investigaciones sobre el conocimiento, las pasiones, la retórica y las bellas artes, a tal punto que, en la historiografía contemporánea bien podríamos referirnos a lo que B. Cummings y F. Sierhuis llaman "el giro afectivo" en los estudios sobre la modernidad temprana. (Cummings y Sierhuis, 2013, pp. 1-9).

32. Una perspectiva "neuro-evolucionista" que reconoce la pertinencia de la historia intelectual de estas preocupaciones se encuentra en (Panksepp \& Biven, 2012, pp. 47-94).

Avicena (Ibn Sina) (2005), "On the Soul”. En: Khalidi, Muhammad Ali (ed.), Medieval Islamic Philosophical Writings, Nueva York, Cambridge University Press, pp. 27-58.

Bartra, Roger (2001), Cultura y melancolía. Las enfermedades del alma en la España del Siglo de Oro, Barcelona, Editorial Anagrama.

Black, Deborah L. (2005), "Psychology: soul and intellect". En: Adamson, Peter, y Taylor, Richard C. (eds.), The Cambridge Companion to Arabic Philosophy, Nueva York, Cambridge University Press, pp. 308-326.

Black, Deborah L. (2014), "The nature of Intellect”. En: Pasnau, Robert (ed.), The Cambridge Companion to Medieval Philo- 
sophy, vol. 1, Nueva York, Cambridge University Press, pp. 320-333.

Bloch, O. René (1971), La philosophie de Gassendi, Países Bajos, Martinus Nijhoff.

Boros, Gábor (2006), "Seventeeth-Century theories of emotion and their contemporary relevance", European Journal of Analytic Philosophy, 2 (1), pp. 125-142, [en línea], disponible en: https://www.ffri.hr/phil/casopis/content/volume_2/ eujap3_boros.pdf [consultado el 30/5/2017]

Burton, Robert (2001), The Anatomy of Melancholy (1621), Nueva York, New York Review Books.

Byers, Sarah (2006), "Life as 'Self-Motion': Descartes and 'The Aristotelians' on the Soul as Life of the Body", The Review of Metaphysics, 59 (4), pp. 723-755.

Conrad, Lawrence I. (1995), "The Arab-Islamic medical tradition". En: Conrad, Lawrence I.; Neve, Michael; Nutton, Vivian; Porter, Roy; Wear, Andrew, The Western Medical Tradition. 800 BC to AD 1800, Nueva York, Cambridge University Press, pp. 93-138.

Cook, Harold J. (1990), "The New Philosophy and medicine in Seventeenth-Century England". En: Lindberg, David C.; Westman, Robert S. (eds.), Reappraisals of the Scientific Revolution, Nueva York, Cambridge University Press, pp. 397-436.

Cook, Harold J. (2002), "Body and Passions: Materialism and the Early Modern State", Osiris, 17, pp. 25-48.

Cottegnies, Line (2002), "Codifying the Passions in the Classical Age: a few reflections on Charles Le Brun's scheme and its influence in France and in England", Études Épistémè, 1, pp. 141-158, [en línea], disponible en: http://www. etudes-episteme.org/2e/spip.php?article12 [consultado el 19/3/2016].

Cummings, Brian y Sierhuis, Freya (eds.) (2013), Passions and Subjectivity in Early Modern Culture, Burlington, Ashgate.

Cyrano de Bergerac (1993), Voyage dans la Lune, París, GF Flammarion.

Cyrano de Bergerac (2003), Les États et Empires du Soleil (1662), París, GF Flammarion.

Damasio, Antonio (1997), El error de Descartes: la razón de las emociones, Santiago de Chile, Andrés Bello.

Davidson, Herbert (1992), Alfarabi, Avicenna, and Averroes on Intellect. Their Cosmologies, Theories of the Active Intellect and Theories of Human Intellect, Nueva York, Oxford University Press.

Descartes, René (1997), Las pasiones del alma, Madrid, Tecnos.

Descartes, René (2000), CEuvres de Descartes, edición Charles Adam y Paul Tannery, París, J. Vrin.
Diderot, Denis (1992), El sueño de D’Alembert (1769), Madrid, Debate.

Duchesneau, François (1973), L'empirisme de Locke, La Haya, Martinus Nijhoff.

Elmer, Peter (1989), "Medicine, religion and the puritan revolution". En: French, Roger; Wear, Andrew (eds.), The Medical Revolution of the Seventeenth Century, Nueva York, Cambridge University Press, pp. 10-45.

Frede, Michael (1987), “On Galen's Epistemology”. En: Frede, Michael, Essays in Ancient Philosophy, Mineápolis, University of Minessota Press, pp. 279-298.

Galeno (2002a), “Que el mejor médico es también filósofo". En: Tratados filosóficos y autobiográficos, Madrid, Editorial Gredos, pp. 81-92.

Galeno (2002b), "Sobre mis propias opiniones". En: Tratados filosóficos y autobiográficos, trad. Teresa M. Manzano, Madrid, Editorial Gredos, pp. 321-357.

Galeno (2003), Sobre las facultades naturales/ Las facultades del alma siguen los temperamentos del cuerpo, Madrid, Editorial Gredos.

Gassendi, Pierre (1959), Exercitationes Paradoxicæ Adversus Aristoteleos (1624)/ Dissertations en forme de paradoxes contre les aristotéliciens, Livres I y II, París, J. Vrin.

Gassendi, Pierre (1964), Disquisitio metaphysica seu dubitationes et instantiae adversus Renati Cartesii metaphysicam et responsa (1644)/ Recherches métaphysiques, ou doutes et instances contre la métaphysique de R. Descartes et ses réponses, París, J. Vrin.

Gert, Bernard (1996), “Hobbes's Psychology”. En: Sorell, T. (ed.), The Cambridge Companion to Hobbes, Nueva York, Cambridge University Press, pp. 157-174.

Hasse, Nikolaus (2014), "The soul's faculties". En: Pasnau, Robert (ed.), The Cambridge Companion to Medieval Philosophy, vol. 1, Nueva York, Cambridge University Press, pp. 305-319.

Hatfield, Gary (1998), "The Cognitive Faculties". En: Garber, Daniel; Ayers, Michael (eds.), The Cambridge History of Seventeenth-Century Philosophy, vol. 2, Nueva York, Cambridge University Press, pp. 953-1002.

Hatfield, Gary (2009), "Psychology in Philosophy: Historical Perspectives". En: Heinämaa, Sara; Reuter, Marina (eds.), Psychology and Philosophy. Inquiries into the Soul from Late Scholasticism to Contemporary Thought, Dordrecht, Springer, pp. 1-25.

Hatfield, Gary (2012), "Mechanizing the Sensitive Soul". En: Manning, Gideon (ed.), Matter and Form in Early Modern Science and Philosophy, Leiden, Brill, pp. 151-186. 
Henry, John (1989), "The matter of souls: medical theory and theology in seventeenth-century England". En: French, Roger; Wear, Andrew (eds.), The Medical Revolution of the Seventeenth Century, Nueva York, Cambridge University Press, pp. 87-113.

Hobbes, Thomas (1997), Leviathan (1651), Nueva York, Cambridge University Press.

Hobbes, Thomas (2000), Tratado sobre el cuerpo (1655), Madrid, Trotta.

Hume, David (2000), A Treatise of Human Nature (1739), editado por D. F. Norton y M. J. Norton, Oxford, Oxford University Press.

Isidoro de Sevilla (2004), Etimologías, Madrid, Biblioteca de Autores Cristianos.

Kemp, Simon, y Fletcher, Garth (1993), "The Medieval Theory of Inner Senses", The American Journal of Psychology, 106 (4), pp. 559-576.

La Mettrie, Julien Offray de (2005), El discurso sobre la felicidad (1748), Buenos Aires, Cuenco de Plata.

La Mettrie, Julien Offray de (2014), El hombre Máquina (1747), El hombre Planta y otros escritos, Buenos Aires, Cuenco de Plata.

Le Brun, Charles (1982), Méthode pour apprendre à dessiner les passions proposée dans une conférence sur l'expression générale et particulière (1702), Nueva York, Reimp. Georg Olms.

LeCoat, Gerard (1971), "Comparative Aspects of the Theory of Expressions in the Baroque Age", Eighteenth-Century Studies, 5 (2), pp. 207-223.

Locke, John (1979), An Essay Concerning Human Understanding (1690), Nueva York, Oxford University Press.

Mandeville, Bernard (2001), La fábula de las abejas (1729), Ciudad de México, Fondo de Cultura Económica.

Manning, Gideon (2008), "Naturalism and Un-Naturalism Among the Cartesian Physicians", Inquiry, 51 (5), pp. 441-463.

Michael, F. y Michael, E. (1990), "The Theory of Ideas in Gassendi and Locke", Journal of the History of Ideas, 51 (3), pp. 379-399.

Norton, David (1981), "The Myth of British Empiricism". History of European Ideas, 1 (4), pp. 331-344.

Nutton, Vivian (1995), "Medicine in Late Antiquity and the Early Middle Ages". En: Conrad, Lawrence I.; Neve, Michael; Nutton, Vivian; Porter, Roy; Wear, Andrew, The Western Medical Tradition. 800 BC to $A D$ 1800, Nueva York, Cambridge University Press, pp. 71-88.

Nutton, Vivian (2004), Ancient Medicine, Londres, Routledge.

Paganini, Gianni (2002), “Hobbes et Gassendi: la psychologie dans le projet mécaniste", Kriterion, 106, pp. 20-41, [En lí- nea] Disponible en: http://www.scielo.br/pdf/kr/v43n106/ v43n106a03.pdf

Panaccio, Claude (2014), "Mental Representation". En: Pasnau, Robert (ed.), The Cambridge Companion to Medieval Philosophy, vol.1, Nueva York, Cambridge University Press, pp. 346-356.

Panksepp, J y Biven, L. (2012), The Archeology of Mind. Neuroevolutionary Origins of Human Emotions, Nueva York, W. W. Norton.

Pintard, René (2000), Le libertinage érudit dans la première moité du XVII siècle, Ginebra, Éditions Slatkine.

Popkin, Richard (1980), The High Road to Pyrrhonism, Indianápolis, Hackett Publishing.

Popkin, Richard (2003), The History of Scepticism. From Savonarola to Bayle, Nueva York, Oxford University Press.

Sarasohn, Lisa T. (1996), Gassendi's Ethics. Freedom in a Mechanistic Universe, Nueva York, Cornell University Press.

Schmidt, Jeremy (2004), "Melancholy and the Therapeutic Language of Moral Philosophy in Seventeenth-Century Thought", Journal of the History of Ideas, 65 (4), pp. 583-601.

Schmitter, Amy M. (2012), "Family Trees: Sympathy, Comparison, and the Proliferation of the Passions in Hume and his Predecessors". En: Pickavé, Martin, y Shapiro, Lisa (eds.), Emotion and Cognitive Life in Medieval and Early Modern Philosophy, Oxford, Oxford University Press, pp. 255-278.

Sorell, Tom (1993), "Seventeenth-Century Materialism: Gassendi and Hobbes". En: Parkinson, G. H. R. (ed.), The Renaissance and Seventeenth-century Rationalism, Nueva York, Routledge, pp. 235-272.

Spinoza, Baruch (2007), Ética demostrada según el orden geométrico (1677), Madrid, Tecnos.

Thomson, Ann (2010), “Animals, Humans, Machines and Thinking Matter, 1690-1707", Early Science and Medicine, 15 (12), pp. 3-37.

Tomás de Aquino (2001a), "Cuestiones disputadas sobre el alma". En: Opúsculos y cuestiones selectas, volumen I, Madrid, Biblioteca de Autores Cristianos, pp. 397-668.

Tomás de Aquino (2001b), Suma de Teología, Tomo I, Madrid, Biblioteca de Autores Cristianos.

Waldow, Anik (2010), "Empiricism and Its Roots in the Ancient Medical Tradition". En: Wolfe, Charles T., y Gal, Ofer (eds.), The Body as Object and Instrument of Knowledge. Embodied Empiricism in Early Modern Science, Dordrecht, Springer, pp. 287-308.

Wear, Andrew (1995), "Medicine in Early Modern Europe, 1500-1700". En: Conrad, Lawrence I.; Neve, Michael; Nut- 
ton, Vivian; Porter, Roy; Wear, Andrew, The Western Medical Tradition. $800 B C$ to $A D$ 1800, Nueva York, Cambridge University Press, pp. 215-361.

Wilson, Catherine (2010), "Matter, Mortality, and the Changing Ideal of Science". En: Sorell, Tom; Rogers, G.A.; Kraye, Jill (eds.), Scientia in Early Modern Philosophy. SeventeenthCentury Thinkers on Demonstrative Knowledge from First Principles, Nueva York, Springer, pp. 35-51.

Wolfe, Charles T. (2010), "Empiricist Heresies in Early Modern Medical Thought". En: Wolfe, Charles T.; Gal, Ofer (eds.),
The Body as Object and Instrument of Knowledge. Embodied Empiricism in Early Modern Science, Dordrecht, Springer, pp. 333-344.

Wright, John P. (1991), "Locke, Willis, and the Seventeenthcentury Epicurean Soul". En: Osler, Margaret J. (ed.), Atoms, Pneuma, and Tranquillity. Epicurean and Stoic Themes in European Thought, Nueva York, Cambridge University Press, pp. 239-258. 